\title{
MS10-03 | Staraniso: Use Of A Webgl-Based 3-D Interactive Graphical Display To Represent And Visualise Data Quality Metrics For Anisotropic Macromolecular Diffraction Data
}

Tickle, lan (Global Phasing Ltd., Cambridge, GBR)

The STARANISO webserver for processing macromolecular diffraction data, either in the unmerged (unscaled or already scaled) state or in the already-scaled and merged state, has been available for use by the community since January 2016, during which time around 6500 user datasets have been successfully processed and the results returned to users. The server is specifically targeted at datasets with significant anisotropic diffraction in the hope that mitigation of the effects of the anisotropy will lead to improved electron-density maps, and this has indeed turned out to be the case for a significant proportion of datasets submitted.

In our view a critical component of the server is a WebGL-based 3-D interactive graphical display to represent and visualise data quality metrics for anisotropically-corrected macromolecular diffraction data. We are of the view that it is impossible to adequately specify the quality of data in terms of a small number of metrics such as completeness, $R_{\text {meas }}$, mean $I / \sigma(I)$ or CC $\left(\frac{1}{2}\right)$, whether specified as spherical bin averages or as overall averages. Equally, even knowledge of the overall anisotropy tensor and associated metrics such as anisotropic diffraction limits do not provide a full picture of the data quality. The 3-D interactive display provided by the server provides full access not only to the anisotropy analysis but also to properties and quality criteria for the dataset, such as the error model, redundancy, completeness and statistical significance. 